\title{
Classification of Sleep Apnea using ECG-Signal Sequency Ordered Hadamard Transform Features
}

\author{
Padmavathi Kora \\ Associate Professor \\ Department of ECE \\ GRIET
}

\author{
Ambika Annavarapu \\ Assistant Professor \\ Department of ECE \\ GRIET
}

\author{
Priyanka Yadlapalli \\ Assistant Professor \\ Department of ECE \\ GRIET
}

\author{
Nagaja Katragadda \\ Assistant Professor \\ Department of ECE \\ GRIET
}

\begin{abstract}
Sleep apnea is a potentially serious breath disorder. This can be detected using a test called as Polysomnography (PSG). But this method is very inconvenient because of its time consuming and expensive nature. This can be overcome by using other methods like Respiratory rate interval, ECG derived respiration and heart rate variability analysis using Electrocardiography (ECG). These methods are used to differentiate sleep apnea affected patients and normal persons. But the major drawback of these is in performance. Hence, in this paper this disadvantage is overcome by considering Sequency Ordered Complex Hadamard Transform (SCHT) as a feature extraction technique. A minute to minute classification of thirty - five patients based on sensitivity, specificity and accuracy are $93.74 \%, 96.15 \%$ and $95.6 \%$.
\end{abstract}

\section{General Terms}

Preprocessing, Feature Extraction, Classifier

Keywords

ECG, SCHT, Sleep Apnea, SVM

\section{INTRODUCTION}

Sleep apnea (SA) is a breathing disorder in which one will experience pauses in breathing or shallow breaths for 10 to 20 seconds more than 30 times per hour during sleep. This disorder leads to other side effects like morning headaches, leg swelling, and day time sleepiness. SA is classified into three classes namely, central SA (CSA), obstructive SA (OSA) and mixed SA. Most patients about $84 \%$ are affected by OSA, because it can be detected neither by blood test nor by any physical change of patient's body. This leads to heart stroke, blood pressure, obesity, arrhythmias, and even diabetes.

Presently, a gold standard for sleep apnea detection is PSG using electroencephalography (EEG), electrooculogram (EOM), ECG, body movement and, nasal airflow. But implementation of PSG experiences practical problems like time consuming and high expense.

Alternative approaches for PSG for apnea detection are developed basing on EEG, ECG, snoring, or pulse oximetry. In them ECG is widely used signal, because feature extraction of ECG signal can be extracted accurately than remaining signals. For example, Bsoul et al. [15] combined RR interval, Yilmaz et al., [16] used feature extraction from a respiratory rate (RR) interval as a classifier input, and introduced such features as inter-quartile range, mean absolute deviation, and median value. and ECG-derived respiration signal (EDR) [17] for real time SA monitoring using a support vector machine (SVM) classification, extracting approximately 111 features in each minute of an ECG segment. Heart Rate Variability (HRV) and wavelet analysis have also been used for ECG initial processing [18, 19]. k-nearest neighbor [20], SVM $[4,21,22]$, and artificial neural network (ATNs) $[18,22]$ are other classification techniques. In this test 12 channels requiring at least 22 wire attachments overnight to a patient to record ECG.

In this paper, alternative SA using Sequency ordered Complex Hadamard Transform (SCHT) as feature extraction is established as shown in Fig??. The features extracted using SCHT are then applied to a minute based Support Vector Machine (SVM) classifier.

\section{SYSTEM MODEL}

ECG data of thirty - five patients are considered for eight hour 12 lead ECG signal is digitized with 12 - bit resolution. Each one minute ECG recording is labeled as 'Ap' and 'No', and placed in three different classes as shown in Table I depending on Apnea - Hypopnea Index (AHI). If AHI of 100 minutes is greater than 15 , the patients are placed in apnea patient class. Class B recordings of time between 5 and 99 minutes contain the AHI index between the range of 5 and 15 . If the apnea minutes are less than 5 the patient will come under normal Class $\mathrm{C}$ patients.

Table I: Classes of Apnea

\begin{tabular}{|l|l|c|}
\hline Class & Name of apnea & $\begin{array}{l}\text { Apnea } \\
\text { Hypopnea Index } \\
\text { (AHI) }\end{array}$ \\
\hline Class A & $\begin{array}{l}\text { Apnea Patient } \\
\text { class }\end{array}$ & $A H I \geq 15$ \\
\hline Class B & $\begin{array}{l}\text { Border line } \\
\text { Class }\end{array}$ & $5 \leq A H I<15$ \\
\hline Class C & $\begin{array}{l}\text { Normal Patient } \\
\text { Class }\end{array}$ & $A H I<5$ \\
\hline
\end{tabular}

\subsection{Preprocessing}

The preprocessing consists mainly of three phases

- $\quad$ Noise removing

- ECG signal segmentation into beats

- Change of non - uniform beats into uniform

The raw ECG is passed through Savizky - Golay FIR smoothing filter to remove noise by using a technique called Linear Least Square (LLS). The noise minimization using LLS is carried out by considering the sum of squared differences between the signal assemblages. These obtained filtered non - uniform signals are segmented into beats detecting the ' $R$ ' peaks. The attained non - uniform samples are resampled to 200 uniform samples. 


\subsection{Feature Extraction}

\subsubsection{Sequency Ordered Complex Hadamard Transform}

Discrete orthogonal transforms are used to make digital systems faster. These are used to transform the characteristics of a signal by altering its domain to understand signal without complexity. An orthogonal transform called BIFORE or HT is represented in (2) is established on Walsh functions by taking only two amplitude levels $\{ \pm 1\}$ forming a rectangular waveform.

A Hadamard matrix, $\mathrm{H}_{\mathrm{M}}$, is defined as a square matrix of dimension $\mathrm{M} \times \mathrm{M}$ in which all elements are ' 1 '. All records of a matrix are $\pm 1,2$. Any two divergent rows of the matrix are orthogonal, that is,

$\mathrm{H}_{\mathrm{M}}^{\mathrm{T}} \mathrm{H}_{\mathrm{M}}=\mathrm{H}_{\mathrm{M}} \mathrm{H}_{\mathrm{M}}^{\mathrm{T}}=\mathrm{NI}_{\mathrm{M}}$

where $\mathrm{H}_{\mathrm{M}}^{\mathrm{T}}$ is the transpose of $\mathrm{H}_{\mathrm{N}}$ and $\mathrm{I}_{\mathrm{M}}$ is the identity matrix of dimension $M \times M$. In reality, a Hadamard matrix is a symmetric matrix whose row and column vectors are orthogonal to each other. The orthogonality property remains unaffected although the row and column vectors or sign of row and column vectors are exchanged as explained by equation (2).

But the major disadvantage of HT is it can be useful only to real - valued signals, but in real time applications complex values are also to be considered. So, Hadamard transforms are replaced by a comprehensive form called CHT, which comprises the values as $\mathrm{C}=\{ \pm 1, \pm \mathrm{j}\}$ where $\mathrm{j}=\sqrt{-1}$. The advantage of CHT is it does not require any additional computational cost because of its complex elements as represented in equations (3) and (4). The orthogonality and simplicity of real HT are not altered because of CHT exclusively dealing with complex - valued signals in DSP applications. CHT is constructed on recursive formula consist of the higher order matrix diagonalising and Kronecker product multiples.

Ordered Hadamard matrices are obtained by organizing the rows of matrices in various ways. Sequency order is chosen in all different orderings as it is more equivalent to Fourier Transform frequency domain representation (3).

SCHT is a discrete orthogonal transform which is made up of the components $\{ \pm 1, \pm \mathrm{j}\}$ and the row vectors of the transform matrix are organized in an ascending order of sequence. Sequency expel to the number of times that the row vector of a matrix crosses the imaginary axis in the unit circle above a normalized time base $0 \leq \mathrm{k} \leq 1$ such that each crossing represent a row of a SCHT matrix. SCHT implementation is modest than DFT in terms of hardware organization and computational convolution

SCHT is implemented by using the extended version of conventional Rademacher functions called as complex Rademacher functions (CRD). CRD are used for both odd and even symmetry though they are incomplete orthogonal set of functions with odd symmetry. CRD is defined as follows:

$$
\operatorname{CRD}(0, k)=g(y)= \begin{cases}1, & k \in\left[0, \frac{1}{4}\right) \\ j, & k \in\left[\frac{1}{4}, \frac{1}{2}\right) \\ -1, & k \in\left[\frac{1}{2}, \frac{3}{4}\right) \\ -j, & k \in\left[\frac{3}{4}, 1\right)\end{cases}
$$

and $\operatorname{CRD}(0, \mathrm{k}+1)=\operatorname{CRD}(0, \mathrm{k})$

where period $\mathrm{K}=1$. Another simpler approach to define CRD over a normalized time base $\mathrm{k} \in[0,1)$ is as follows:

$$
\operatorname{CRD}(0, \mathrm{k})=\mathrm{e}^{-\frac{\mathrm{j} \pi \operatorname{csgn}\left(\mathrm{e}^{\mathrm{j} 2 \pi \mathrm{t}}\right)}{\sqrt{2}}}
$$

which maps the real-valued $t$ to the element from the set $\{ \pm 1, \pm j\}$ where $\operatorname{csgn}(y)$ is defined for any nonzero complex number $\mathrm{y}=\mathrm{y}_{1}+\mathrm{jy}_{2}$ as follows:

$\operatorname{csgn}(y)=\operatorname{sgn}\left(y_{1}\right)+\operatorname{jsgn}\left(y_{2}\right)$

where $\operatorname{sgn}(\cdot)$ represents the signum function. But if $\mathrm{y}$ is a real number, it can be shown that $\operatorname{csgn}(y)=\operatorname{sgn}(y)$.

Real Rademacher functions are partially introduced by the complex element $\mathrm{j}$ where $\mathrm{j}=\sqrt{-1}$ to compress the orthogonality property of the Rademacher functions. For nonnegative integer $\mathrm{p}$, the complex Rademacher function series can be defined as follows:

$$
\operatorname{CRD}(\mathrm{p}, \mathrm{k})=\operatorname{CRD}\left(0,2^{\mathrm{p}} \mathrm{k}\right)
$$

where $\mathrm{p}=0,1, \ldots \mathrm{m}-1$. By constricting $\operatorname{CRD}(0, \mathrm{k})$ in the horizontal direction by a factor of $2^{p}$ results in $\operatorname{CRD}(\mathrm{p}, \mathrm{k})$. By discretizing the CRD function series the CRD matrices are obtained i.e., sampling the CRD function series at $\mathrm{M}=2^{\mathrm{m}}$ equally spaced locations within the normalized time $k$. Let $R_{m}$ be the CRD matrix of dimension $\mathrm{m} \times 2 \mathrm{~m}$.

Then, the $\mathrm{p}^{\text {th }}$ row of $\mathrm{R}_{\mathrm{m}}$ is defined as

$\mathrm{R}_{\mathrm{m}}(\mathrm{p}, \mathrm{l})=\operatorname{CRD}\left(\mathrm{r}, \frac{\mathrm{l}}{2^{\mathrm{m}}}+\frac{1}{2^{\mathrm{m}+2}}\right)=\operatorname{CRD}\left(\mathrm{p}, \frac{4 \mathrm{l}+1}{2^{\mathrm{m}+2}}\right)(8)$

where $\mathrm{p}=0,1, \ldots, \mathrm{m}-1$ and $\mathrm{l}=0,1, \ldots, 2 \mathrm{~m}-1$.

Let us, as an example, consider $M=8$. Then, the $3 \times 8$ complex Rademacher matrix is fashioned in (9) by placing (3) and (4) in (8) and attained values are tabulated in TABLE II.

TABLE II. Complex Rademacher matrix values for $\mathbf{M}=\mathbf{8}$

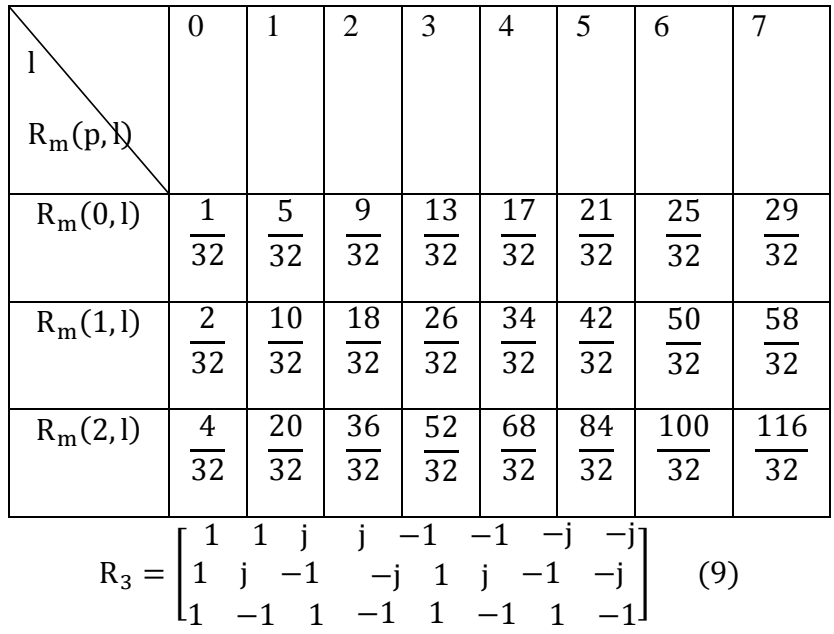

Using complex Rademacher matrices, the SCHT matrices are created based on the products of the row vectors of complex Rademacher matrices. Let $H_{M}$ be the SCHT matrix of size $M \times M$ where $M=2^{m m}$. Then, it is defined as 


$$
\mathrm{H}_{\mathrm{M}}(\mathrm{g}, \mathrm{l})=\prod_{\mathrm{p}=0}^{\mathrm{m}-1} \mathrm{R}_{\mathrm{m}}(\mathrm{p}, \mathrm{l})^{\mathrm{b}_{\mathrm{p}}}
$$

where $R_{m}(p, l)$ is the $\left(p^{\text {th }}, l^{\text {th }}\right)$ element of the complex Rademacher matrix,

$$
\mathrm{g}=\mathrm{b}_{\mathrm{m}-1} 2^{\mathrm{m}-1}+\cdots+\mathrm{b}_{1} 2^{1}+\mathrm{b}_{0} 2^{0}
$$

and $b_{p}=0$ or 1 . Let $R_{m}(p)$, for $p=0,1 \ldots m-1$, be the $p^{\text {th }}$ row vector of the complex Rademacher matrix. For example, let us consider $\mathrm{H}_{8}$ (12) which can be achieved by using (10) and the values are tabulated in TABLE II. Equation (13) is obtained by substituting equation (3) in (12).

Table III. SCHT matrix $\mathrm{H}_{8}(\mathrm{~g}, \mathrm{l})$ for $\mathrm{M}=8$

\begin{tabular}{|c|c|}
\hline $\mathrm{G}$ & $\mathrm{H}_{8}(\mathrm{~g}, \mathrm{l})$ \\
000 & 1 \\
001 & $\mathrm{R}_{3}(0,1)$ \\
010 & $\mathrm{R}_{3}(1,1)$ \\
011 & $\mathrm{R}_{3}(1,1) \odot \mathrm{R}_{3}(0,1)$ \\
100 & $\mathrm{R}_{3}(2,1)$ \\
101 & $\mathrm{R}_{3}(2,1) \odot \mathrm{R}_{3}(0,1)$ \\
110 & $\mathrm{R}_{3}(2,1) \odot \mathrm{R}_{3}(1,1)$ \\
111 & $\mathrm{R}_{3}(2,1) \odot \mathrm{R}_{3}(1,1) \odot \mathrm{R}_{3}(0,1)$ \\
\hline
\end{tabular}

$\mathrm{H}_{8}(\mathrm{~g}, 1)=\left[\begin{array}{c}1 \\ \mathrm{R}_{3}(0,1) \\ \mathrm{R}_{3}(1,1) \\ \mathrm{R}_{3}(1,1) \odot \mathrm{R}_{3}(0,1) \\ \mathrm{R}_{3}(2,1) \\ \mathrm{R}_{3}(2,1) \odot \mathrm{R}_{3}(0,1) \\ \mathrm{R}_{3}(2,1) \odot \mathrm{R}_{3}(1,1) \\ \mathrm{R}_{3}(2,1) \odot \mathrm{R}_{3}(1,1) \odot \mathrm{R}_{3}(0,1)\end{array}\right]$

$\mathrm{H}_{8}(\mathrm{~g}, \mathrm{l})=\left[\begin{array}{cccccccc}1 & 1 & 1 & 1 & 1 & 1 & 1 & 1 \\ 1 & 1 & \mathrm{j} & \mathrm{j} & -1 & -1 & -\mathrm{j} & -\mathrm{j} \\ 1 & \mathrm{j} & -1 & -\mathrm{j} & 1 & \mathrm{j} & -1 & -\mathrm{j} \\ 1 & \mathrm{j} & -\mathrm{j} & 1 & -1 & -\mathrm{j} & \mathrm{j} & -1 \\ 1 & -1 & 1 & -1 & 1 & -1 & 1 & -1 \\ 1 & -1 & \mathrm{j} & -\mathrm{j} & -1 & 1 & -\mathrm{j} & \mathrm{j} \\ 1 & -\mathrm{j} & -1 & \mathrm{j} & 1 & -\mathrm{j} & -1 & \mathrm{j} \\ 1 & -\mathrm{j} & -\mathrm{j} & -1 & -1 & \mathrm{j} & \mathrm{j} & 1\end{array}\right]$

The positions of binary ones of decimal value $g$ bring into being by the row index values 2,1 and 0

\subsection{Classifier}

Classification of apnea and non - apnea minute based ECG recording is studied by SVM with a Gaussian radial basis function kernel. In classification phase, performance of SVM is checked using a 10 - fold cross validation (CV) scheme. In this set of samples are divided randomly into 10 approximately balanced and equal parts. At each time these subsets are excluded from total samples and remaining samples are set for training. Percentage of correctly classified data by 10 - fold CV accuracy. 10-fold CV was also used to optimize two Gaussian kernel parameters during the learning phase: $\gamma$, the width of the Gaussian kernel and $\sigma$, which controls the trade-off between minimizing training errors and model complexity. In this proposed system, SVM classifier performance is evaluated on the test set using accuracy, sensitivity, and specificity defined as follows.

$$
\begin{aligned}
& \text { specificity }=\frac{\text { True }_{\text {Negative }}}{\text { True }_{\text {Negative }}+\text { False }_{\text {Positive }}} \times 100 \\
& \text { sensitivity }=\frac{\text { True }_{\text {Positive }}}{\text { True }_{\text {Positive }}+\text { False }_{\text {Negative }}} \times 100 \\
& \text { Accuracy }=\frac{T P+T N}{T P+T N+F P+F N} \times 100 \\
& \text { TP } \left.\text { (True }_{\text {Positive }}\right) \\
& =\text { Count of all the correctly classified Normal beats } \\
& \text { TP }\left(\text { True }_{\text {Negative }}\right) \\
& =\text { Count of all the correctly classified Abnormal beats } \\
& \text { FP (False } \text { Positive }) \\
& =\text { Count of Normal beats classified as Abnormal beats } \\
& \text { FN (False } \text { Negative }) \\
& =\text { Count of Abnormal beats classified as Normal beats }
\end{aligned}
$$

\begin{tabular}{|c|c|c|c|}
\hline \multirow[t]{2}{*}{ Approach } & \multicolumn{3}{|c|}{ Performance } \\
\hline & Sensitivity & Specificity & $\begin{array}{l}\text { Acc } \\
\text { urac } \\
y\end{array}$ \\
\hline $\begin{array}{l}\text { Measure of } \\
\text { minutes of sleep } \\
\text { disordered } \\
\text { respiration [1] }\end{array}$ & NA & NA & 91 \\
\hline $\mathrm{SaO}_{2}[2]$ & 90.1 & 82.9 & NA \\
\hline $\mathrm{SaO}_{2}$ and EEG [3] & 91 & 83.3 & 88.5 \\
\hline $\mathrm{SpO}_{2}$ and ECG[4] & 79.75 & 85.89 & 84.40 \\
\hline EEG [5] & 69.64 & 44.44 & NA \\
\hline ECG [6] & NA & NA & 92.67 \\
\hline $\begin{array}{l}\text { Fourier and } \\
\text { Wavelet transform } \\
\text { of HRV [7] }\end{array}$ & 90.8 & NA & NA \\
\hline $\begin{array}{l}\text { Bivariate } \\
\text { autoregressive } \\
\text { model of HRV [8] }\end{array}$ & NA & NA & 85 \\
\hline
\end{tabular}

\section{RESULTS \& DISCUSSION}

Based on various records of Apnea - ECG database efficiency of proposed method is calculated in terms of specificity and sensitivity in addition to the accuracy using the MATLAB tool for classification. Table IV represents the comparison of our proposed method with the previous methods.

Table IV Comparison of Proposed technique with previous techniques 


\begin{tabular}{|c|l|l|l|}
\hline $\begin{array}{c}\text { RR interval based } \\
\text { Classification [9] }\end{array}$ & NA & NA & 89 \\
\hline ECG - SVM [10] & 92.9 & 100 & 96.5 \\
\hline RI Waveform [11] & 88.1 & 65.7 & 91.2 \\
\hline $\begin{array}{c}\text { Nasal Pressure - } \\
\text { ECG [12] }\end{array}$ & 75 & 100 & 87.5 \\
\hline $\begin{array}{c}\text { W - ECG with } \\
\text { features [13] }\end{array}$ & 81.37 & 79.09 & 80.14 \\
\hline $\begin{array}{c}\text { W - ECG + PCA } \\
\text { [14] }\end{array}$ & 95.20 & 92.65 & 93.91 \\
\hline $\begin{array}{c}\text { Proposed Method } \\
\text { Prod }\end{array}$ & 93.74 & 96.15 & 95.6 \\
\hline
\end{tabular}

\section{CONCLUSION}

In this paper, we studied the ECG signal based sleep apnea detection and, further developed a novel form using SCHT as feature extraction and calculated the effectiveness in terms of sensitivity, specificity and accuracy. From the experimental results we conclude that the SCHT get the best accuracy than the previous methods.

In future we plan to enhance the performance of feature extraction using optimization, and then make it to work in real - time monitoring system and analyze the ECG signal of patients during sleep.

\section{ACKNOWLEDGMENTS}

Our thanks to the Gokaraju Rangaraju Institute of Engineering \& Technology, Hyderabad, India

\section{REFERENCES}

[1] P. Chazal, T. Penzel and C. Heneghan,"Automated Detection of Obstructive Sleep Apnoeaa at Different Time Scales Using the Electrocardiogram," Institute of Physics Publishing, vol. 25, no. 4, pp.967-983, Aug. 2004.

[2] D. Alvarez, R. Hornero, D. Abasolo, F. Campo and C. Zamarron, "Nonlinear Characteristics of Blood Oxygen Saturation from Nocturnal Oximetry for Obstructive Sleep Apnoea Detection," Institute of Physics Publishing, vol. 27, no. 4, pp. 399-412, Apr. 2006.

[3] D. Avarez, R. Hornero, J. Marcos, F. Campo and M. Lopez, "Spectral Analysis of Electroencephalogram and Oximetric Signals in Obstructive Sleep Apnea Diagnosis," in Proceedings of the 31st IEEE International Conference on Engineering in Medicine and Biology Society (EMBS 2009), pp. 400-403, Sep. 2009.

[4] B. Xie, H. Minn, "Real Time Sleep Apnea Detection by Classifier Combination," in IEEE Transactions on Information Technology in Biomedicine (in Press), 2012.

[5] R. Lin, R. Lee, C. Tseng, H. Zhou, C. Chao, J. Jiang, “A New Approach for Identifying Sleep Apnea Syndrome Using Wavelet Transform and Neural Networks," Biomedical Engineering: Applications, Basis \& Communications, vol. 18, no. 3, pp. 138-143, 2006.

[6] Q. Manrique, A. Hernandez, T. Gonzalez, F. Pallester and C. Dominquez, "Detection of Obstructive Sleep Apnea in ECG Recordings Using Time-Frequency
Distributions and Dynamic Features," in Proceedings of the IEEE International Conference on Engineering in Medicine and Biology Society( EMBS 2009), pp. 55595562, Sep. 2009.

[7] M. Schrader, C. Zywietz, V. Einem, B. Widiger, G. Joseph, "Detection of Sleep Apnea in Single Channel ECGs from the PhysioNet Data Base," Computers in Cardiology 2000, vol. 27, pp. 263-266, Sep. 2000.

[8] M. Mendez, D. Ruini, O. Villantieri, M. Matteucci, T. Penzel and A. Bianchi, "Detection of Sleep Apnea from Surface ECG Based on Features Extracted by an Autoregressive Model, " in Proceedings of the IEEE International Conference on Engineering in Medicine and Biology Society (EMBS 2007), pp. 6105-6108, Aug. 2007.

[9] B. Yilmaz, M. Asyali, E. Arikan, S. Yektin and F. Ozgen, "Sleep Stage and Obstructive Apneaic Epoch Classification Using Single- Lead ECG," in Biomedical Engineering Online, vol. 9, 2010.

[10] Laiali Almazaydeh, Khaled Elleithy, Miad Faezipour, "Obstructive Sleep Apnea Detection Using SVM-Based Classification of ECG Signal Features", 34th Annual International Conference of the IEEE EMBS San Diego, California USA, 28 August - 1 September, 2012

[11] A. Thommandram, J.M. Eklund and C. Mcgregor, Detection of apnoea from respiratory time series data using clinically recognizable features and $\mathrm{kNN}$ classification, 35th Annual Internatioal Conference of IEEE Engineering in Medicine and Biology Society (2013), 5013-5016.

[12] J.L. Pepin, P. Defaye, S. Christophle-Boulard, T.R. Tamisier and P. Levy, Sleep apnea diagnosis using an ECG holter device including a nasal pressure (NP) recording: Validation of visual and automatic analysis of nasal pressure versus full polysomnography, Sleep Medicine 10 (2009), 651-656.

[13] J. Gubbi, A. Khandoker and M. Palaniswami, Classification of sleeptypes using wavelet packet analysis of short-term ECG signals, J. Clin. Monit. Comput. 26 (2012), 1-11.

[14] Vega Pradana Rachim, Gang Li and Wan-Young Chung, "Sleep apnea classification using ECG-signal waveletPCA features", in Bio-Medical Materials and Engineering 24 (2014) 2875-2882

[15] M. Bsoul, H. Minn and L. Tamil, Apnea medassist: Realtime sleep apnea monitor using single-lead ECG, IEEE Transactions on Information Technology in Biomedicine 15 (2011), 416-427.

[16] B. Yilmaz, M. Asyali, E. Arikan, S. Yektin and F. Ozgen, Sleep stage and obstructive apneaic epoch classification using single-lead ECG, Biomedical Engineering Online 9 (2010), 1-14,.

[17] C. Avci, I. Delibasoglu and A. Akbas, Sleep apnea detection using wavelet analysis of ECG derived respiratory signal, International Conference on Biomedical Engineering (2012), 272-275.

[18] G. Sannino, I. De Falco and G. De Pietro, An automatic rules extraction approach to support OSA events detection in a mHealth system, IEEE Journal of Biomedical and Health Informatics (2014), will be 
published.

[19] A. Thommandram, J.M. Eklund and C. Mcgregor, Detection of apnoea from respiratory time series data using clinically recognizable features and $\mathrm{kNN}$ classification, 35th Annual Internatioal Conference of IEEE Engineering in Medicine and Biology Society (2013), 5013-5016.

[20] Y. Ke, L. Chen, F. Lan, Y. Jia, P. Li, X. Zhao, H. Qi, P. Zhou, L. Zhang, B. Wan and D. Ming, Visual attention recognition based on nonlinear dynamical parameters of EEG, Bio-Medical Material Engineering 24 (2014), 349355.
[21] G. Li and W.Y. Chung, Detection of driver drowsiness using wavelet analysis of heart rate variability and a support vector machine classifier, Sensors 13 (2013), 16494-16511. V.P. Rachim et al. / Sleep apnea classification using ECG-signal wavelet-PCA features 2881

[22] A. H. Khandoker, J. Gubbi and M. Palaniswami, Automated scoring of obstructive sleep apnea and hypopnea events using short-term electrocardiogram recordings, IEEE Transactions on Information Technology in Biomedicine (2009), 1057-1067. 